\title{
Who should wear a face mask? Experts weigh in on Canada's COVID-19 response
}

\author{
— Cite as: CMAJ 2020 April 20;192:E440-1. doi: 10.1503/cmaj.1095863
}

Posted on cmajnews.com on April 3, 2020

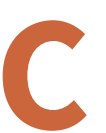
anadians are pushing for more information about the trajectory of the severe acute respiratory syndrome coronavirus 2 (SARS-CoV-2) pandemic and increasingly questioning official advice, especially when it comes to recommendations against wearing face masks. To put these developments in perspective, CMAJ reached out to infectious disease specialists Dr. Srinivas Murthy of BC Children's Hospital and the University of British Columbia in Vancouver, Dr. Matthew Oughton of Jewish General Hospital and McGill University in Montreal, and Dr. Alon Vaisman of the University Health Network in Toronto.
\end{abstract}

\section{CMAJ: Which recent developments} related to Canada's response to the pandemic have most concerned or encouraged you?

Oughton: The most concerning development this week has been the acknowledgement by several provinces that our supplies of personal protective equipment (PPE) are running low... Our federal government needs to develop and maintain domestic manufacturing capacity for these essential products.

One encouraging development this week has been the several reports on effective means of sterilizing N95 respirators, making them safe for reuse. Although not a long-term solution, this may help Canadian health care facilities get more use from existing stocks while new supplies are being shipped and manufactured.

Vaisman: The fact that Canada, in the first place, was not a self-sustaining nation for medical equipment is the most concerning aspect of the federal response. A nation with such wealth, education, natural resources, and manufacturing capacity should not have to rely on foreign nations to supply its most vital equipment. Now Canadian manufacturers are scrambling to produce devices and PPE for our front-line staff.

Murthy: The trajectories [of outbreaks] in the United States are obviously concerning and some of their mitigation strategies are likely inadequate at this point. Given our proximity economically, geographically and emotionally, that's a huge target for us to keep our eye on. [Encouragingly, however] British Columbia released modelling data showing the impact of physical distancing... Although the case count continues to rise [in $\mathrm{BC}$ ], a lack of physical distancing would have led to a faster rise than what we're seeing.

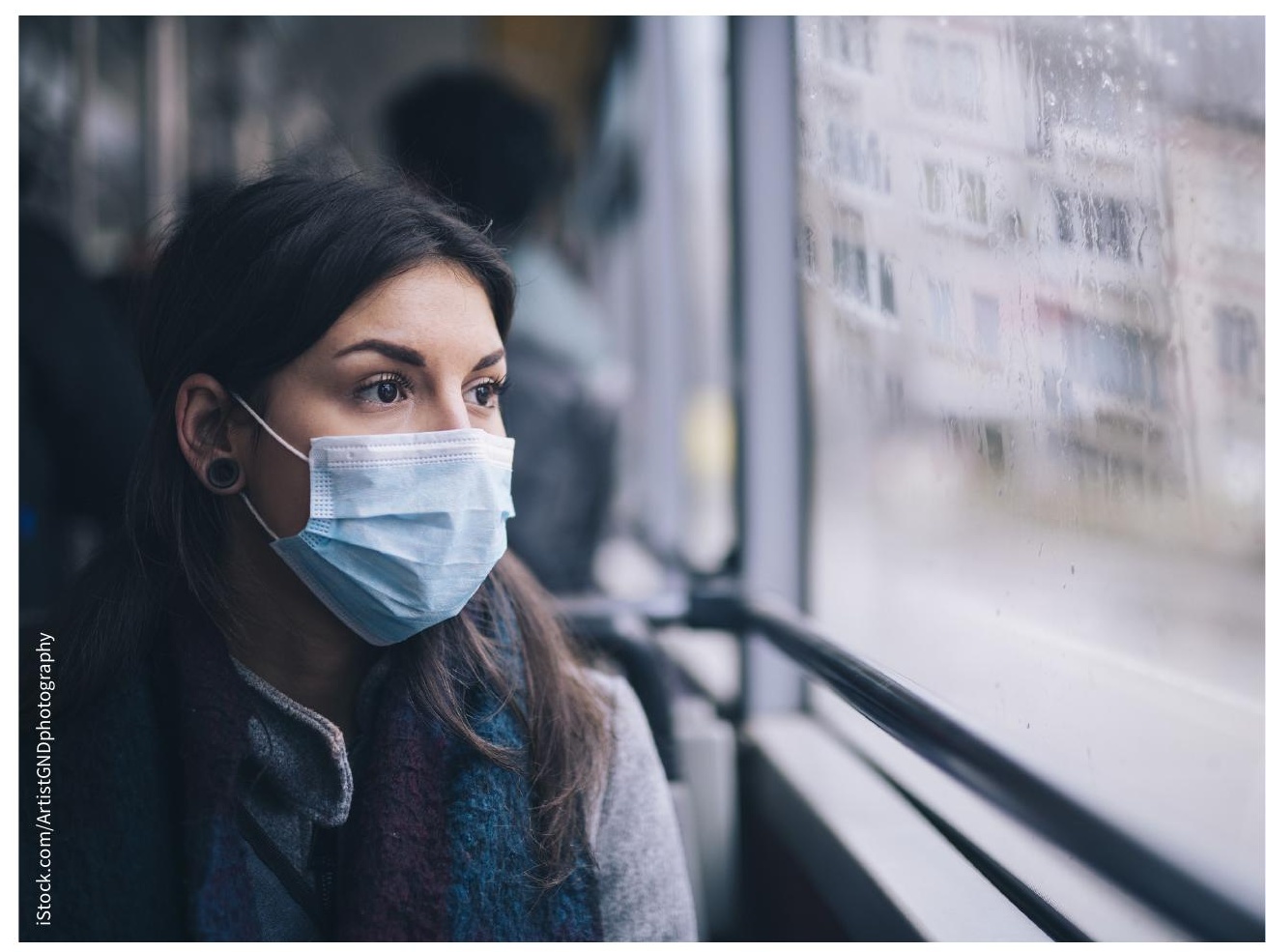

Debate over who should wear face masks has intensified as supplies have run low.
CMAJ: Some health experts, including officials from Asian countries, have argued that Western countries are making a mistake by discouraging the general public from wearing face masks in public. Canadian officials have argued, however, that masks won't protect healthy people and should be reserved to protect health workers. Who should wear a mask?

Oughton: Most people agree that N95 respirators should be reserved for use in medical facilities. As for procedural masks, the ferocity of the debate seems matched only by the absence of compelling evidence to support either side. If supplies of masks were not limited, an argument could be made that public 
masking may provide some degree of benefit... but in the [context] of limited supplies it makes sense to prioritize delivery of masks to high-risk settings, such as hospitals and outpatient clinics.

Murthy: There is also the argument that wearing a mask, if you are not used to wearing one, could lead you to touch your face more. And then there's the question of what kinds of face masks to use. Homemade ones will not protect you much, but they might do something. The surgical face masks are a bit more protective, and the $\mathrm{N} 95$ respirators will protect you against almost everything, but only if you wear them correctly and only for a short period of time. With all those caveats, it's difficult to say what people in the community should wear... At some point, you're going to get diminishing returns based on the risk of exposure being vanishingly small.

\section{CMAJ: Reports from the United States} and Iceland suggest that as many as $25 \%$ to $50 \%$ of people with COVID-19 do not have symptoms but may still spread the virus. What different strategies will be necessary if many cases are shown to be asymptomatic?

Oughton: There have been several studies recently on pre-symptomatic and asymptomatic transmission, including a recent report that cited pre-symptomatic transmission as a cause of about $6 \%$ of communityacquired cases [in Singapore]. As we are understanding more about the epidemiology and the transmission dynamics of SARS-CoV-2, it becomes increasingly clear that physical distancing is an important strategy for everyone to follow, as even fully asymptomatic people may pose a risk of disease transmission.

Murthy: We need to understand more about what asymptomatic carriers can do in terms of the viral dynamics in a population... You really want to make sure it's real before committing to policies. If there is a significant burden of asymptomatic transmission, then effectively everyone will have to be socially isolated almost completely.

Vaisman: Stricter enforcement may be needed (fines, curfews, reduction in public transportation). Also, masking of the public may [then] be beneficial, if this is in fact accurate.

CMAJ: The United States and some provinces have shared modelling predictions of how many people might die during the pandemic. However, Prime Minister Justin Trudeau has not yet commented on how many deaths might occur in Canada or how long physical distancing measures might last. How important is it for the government to be transparent about possible outcomes of the pandemic?

Vaisman: I think that transparency is very important, and that people should understand what the experts are saying. These predictions must be qualified with their limitations and degrees of uncertainty. They must also be provided in context of what we don't know about the virus and what has already been done to contain the virus.

Murthy: There's a fine line between scaring the public into listening to you versus working with them, understanding that there are gaps in knowledge and we're trying to figure this out together... Seeing both the best- and worst-case scenarios has its uses but also has its harms in terms of the fear and the stigma that might get attached to people who have symptoms.

Oughton: There is a risk of having some of the audience "tune out" if they do not enjoy the message they are hearing. On the other hand, providing the best information we have on likely outcomes of COVID-19 would be very helpful for the public in understanding the priorities and plans being used by our government. Overall, I would prefer that Mr. Trudeau be transparent and make the information public and accessible to all Canadians.

CMAJ: There's been a lot of buzz about the possibility of using contact tracing apps to support a more flexible approach to physical distancing. What do you think about these apps?

Oughton: Contact tracing apps and metadata analysis can be useful... as they may identify many contacts that would otherwise be untraceable. Clearly, this technology comes at the expense of individual privacy, so apps like this need to be used for clearly specified public health purposes in conjunction with tight legislative control to prevent "mission creep" and unauthorized use.

Murthy: Ensuring the public is on board with such a thing before it's rolled out should be a priority - explaining what they're doing, why they're doing it, what's being collected and why it's being collected - instead of just doing it... The pandemic is a very large imposition on people's lives, so anything that would shorten it may be worth sacrificing some degree of privacy along the way. Canadians should be able to make that decision.

Lauren Vogel, CMAJ 